\title{
La Deserción Estudiantil en el Programa de Ingeniería de Sistemas de la Univer- sidad de la Amazonia (2012-i a 2015-i): Una Lectura Institucional y Antropológica del Asunto
}

\author{
Dropout in the Systems Engineering Program at the University of \\ the Amazon (2012 -I 2015 -I) : an institutional and anthropological \\ reading topic
}

Verenice Sánchez Castillo

Ingeniera Agroecóloga, MSc. en Ambiente y Desarrollo, Est. de Doctorado en Antropología. Docente Programa Ingeniería Agroecológica de la Universidad de la Amazonia. Grupo de Investigación en Agroecología y Desarrollo Rural (GIADER).

Correo: ve.sanchez@udla.edu.co
Carlos Alberto Gómez Cano Contador Público, Administrador Público, Esp. en Pedagogía y en Gestión Pública, Est. Maestría en Ciencias de la Educación. Docente del Programa de Contaduría Pública de la Universidad de la Amazonia. Grupo de Estudios de Futuro en el Mundo Amazónico (GEMA). Correo carlosgomez325@gmail.

Mayra Alejandra Gaviria Alvarado

Ingeniera Agroecóloga, Esp. en Pedagogía.

Integrante del Grupo de Investigación en

Agroecología y Desarrollo Rural (GIADER).

Correo: malac06mayra@gmail.com 


\section{Resumen}

L as políticas para el fortalecimiento de la educación superior, usual- Palabras clave: mente han estado dirigidas a aumentar la capacidad de cobertura de cada uno de los programas académicos, lo que se ha venido logrando paulatinamente. Sin embargo, la situación parece no ser tan ventajosa cuando se mantiene de manera constante los altos niveles de deserción estudiantil, siendo preocupante no tener claridades alrededor de las causales y así tomar decisiones que desencadenen en estrategias de mejora y atención a la problemática. La presente investigación tomando como estudio de caso el Programa de Ingeniería de Sistemas de la Universidad de la Amazonia, hizo un abordaje de tipo mixto empleando técnicas como arqueo de archivo, encuestas, observación estructurada y entrevistas, aplicadas a los desertores hizo una lectura sobre las causales de su abandono en el Programa. Al respecto, encontró que aunque como en los diferentes estudios de deserción aparecen factores como la dificultad económica o esta no era mi carrera, la situación es mucho más compleja y está relacionada con la vocación y el imaginario de vida que tienen los estudiantes.

\footnotetext{
Abstract

$\mathrm{P}$ olicies for the strengthening of higher education have usually been aimed at increasing the covering capacity of each degree program, which has been gradually achieved. However, the situation seems not dropout, education, factors to be so advantageous when consistent high rate levels of dropout are held, being worried about not having clear answers about the causes and thus forced to make decisions that trigger strategies looking for improvement bringing attention to the problem. This research taken as a case study of the Systems Engineering Program at the University of the Amazon, tackled it as a mixed approach using techniques such as balance file, surveys, observation and structured interviews, applied to deserters gave a reading on causes for abandoning the program. In this regard, it was found that as in other desertion studies regretting factors such as economic hardship or this was not the career I should have chosen, the situation is much more complex and is related to the vocation and imaginary life that students have shown.

\section{ginarios}




\section{Introducción \\ El concepto de deserción escolar}

Tinto [1] expone, desde una percepción individual, que desertar está relacionado con el fracaso para completar un determinado curso de acción o alcanzar un objetivo trazado en pos del cual ingresó un estudiante al sistema universitario. Igualmente, sostiene que la deserción involucra procesos sociales e intelectuales a través de los cuales las personas proyectan las metas deseadas. En el mismo sentido, Buentello [2] sostiene que la deserción es el abandono de las obligaciones académicas y de la concurrencia a los espacios de formación, refriéndose especialmente a los estudiantes que dejan de asistir a clases y desaparecen del sistema educativo.

Las causas del fenómeno, según Silva [3], están relacionadas con inasistencia, reprobación o vencimiento de los cursos matriculados. Sin embargo, Lopera [4] realizó un estudio en la Universidad del Rosario, el cual le permitió concluir que deserción estudiantil no es solo importante por la inasistencia de los estudiantes a sus jornadas educativas, sino que es un tema de interés dentro de las políticas educativas a nivel nacional e institucional debido a los efectos que este tema tiene sobre la movilidad social y las restricciones presupuestales de las instituciones educativas.

Para Bravo \& Mejía [5], el problema de la deserción estudiantil se ha convertido en una problemática que afecta el óptimo funcionamiento de las universidades, pues genera desequilibro en los procesos de planeación académicos y administrativos. La deserción estudiantil es una pro- blemática multicausal, pues incluye variables como: actores públicos y privados, estudiantes, familias y al Estado; además, es multidimensional pues tiene implicaciones académicas, financieras, socioeconómicas, psicológicas y sociales [6].

Marina [7] afirma que el abandono escolar es una preocupación de orden mundial, además sostiene que es un claro indicador de la situación educativa de la nación, específicamente de las políticas y estrategias asumidas para su atención. Por otro lado, Silva [8] sostiene que la deserción escolar debe ser vista y reconocida como un problema que trasciende del campo educativo al social, pues trae consigo una serie de factores asociados que van más allá de los límites de la educación.

Los indicadores de deserción tienen sentidas variaciones dependiendo del país donde se analicen. Roa [9] afirma que la Organización para la Cooperación y el Desarrollo Económico (OCDE) reveló, en 2012, importantes datos sobre la deserción en el mundo. El estudio colocó a México y Turquía en los primeros lugares con tasas de deserción universitaria que rondaban el 38\%; Suecia registró un indicador del $36 \%$ y Portugal del 31\%. En los últimos lugares, donde se considera que los niveles de deserción son mínimos, se encuentran Alemania con un 4.3\%, Países Bajos con un $0.7 \%$ y Finlandia con un $0.45 \%$. Igualmente, el autor sostiene que los factores con mayor incidencia en la deserción universitaria están relacionados con la situación económica, pérdida de asignaturas, embarazo, inconvenientes familiares, horarios y falta de flexibilidad curricular.

Las tesis sobre los factores que inciden en la deserción universitaria son abundantes, toda vez que responden a contex- 
tos, situaciones y políticas diferentes. Por ejemplo, Rojas [10] sostiene que de manera tradicional la deserción universitaria se ha asociado con presiones económicas, académicas, laborales e incluso psicológicas, no obstante, aduce que, en el contexto actual de nuestro mundo globalizado, la persistencia e incremento significativo del fenómeno de la deserción universitaria puede referir a una profunda crisis social en la valoración de la educación.

Finalmente, sea cual sea el factor asociado a la deserción universitaria, lo único realmente estandarizado es que es una preocupación de orden mundial. Cada país lucha con sus condiciones, conflictos y dificultades por la erradicación de este fenómeno que, sin importar el contexto en que se genere, está arrebatando a miles de personas de los brazos de la formación profesional en las universidades. En palabras de la CEPAL [11], los gobiernos deben dar prioridad en sus agendas sociales a este suceso, sin embargo, expresa que los esfuerzos por reducir la deserción deberán estar acompañados de estrategias para la generación de empleos de calidad y una adecuada política de enganche laboral.

El MEN [6] sostiene que la investigación sobre la deserción en Colombia hasta hace unos años eran relativamente escasa, más aún pensar en políticas y reformas educativas. Este problema es aducido al trabajo individualizado de las universidades, donde solo se realizaban análisis endógenos. Igualmente, afirma que el país, antes del 2003, carecía de un marco conceptual solido que soportara las intervenciones en el sector y que permitiera la generación de estrategias para la mitigación del fenómeno.

Salcedo [12] expone que una de las formas más prácticas para analizar la deserción es la matemática. Sostiene que esta puede ser definida como "comparación numérica entre la matrícula inicial menos el número de egresados de último año". Este planteamiento conlleva a un análisis por cohortes, lo cual genera un gran debate alrededor de los estudiantes que no logran finalizar su proceso de formación académica en el nivel profesional. Sobre el particular, el MEN sostiene que la deserción promedio por cohortes, desde 1998 hasta 2006, fue de $45.3 \%$, una cifra que a todas luces muestra un panorama desolador para el sistema educativo, pues poco más de la mitad de los estudiantes lograron obtener su título profesional universitario.

El anterior escenario es bastante árido. Aunado a ello, los estudios posteriores no son muy promisorios en términos de reducción del fenómeno. La Revista Dinero [13] afirma que recientes estudios de reconocidas universidades en el país sostienen que la deserción estudiantil en las universidades colombianas está sobre el 48\%, una cifra bastante desalentadora si se tienen en cuenta los esfuerzos realizados por las universidades y las instituciones como el ICETEX para el fomento de la permanencia. Igualmente, afirma que los problemas más comunes están relacionados con los costos de las matrículas, los altos costos de la manutención de los estudiantes, la falta de preparación con la que salen de los colegios y problemas familiares.

La deserción parece ser una pandemia educativa, pues sus efectos no se detienen y, por el contrario, son cada vez más nocivos para el sistema educativo, máxime en un país como Colombia, donde los conflictos armados han generado un gran desequilibrio social y, por tanto, la formación universitaria se convierte en una de 
las pocas estrategias para arrebatar jóvenes de los brazos de la violencia. Según el MEN (2013), la deserción en el nivel universitario alcanzó el $44.9 \%$ durante el año 2013, teniendo los grandes picos en la formación técnica con un $62.4 \%$ y la tecnológica con un 53.8\%. Igualmente, se afirma que los mayores impactos del fenómeno se concentran en los cuatro primeros semestres de la carrera, en los cuales se produce el $75.1 \%$ de la deserción por cohorte de estudiantes del nivel universitario. Los altos niveles de deserción en el país son preocupantes, máxime cuando en los últimos años el sistema educativo de educación superior se ha esforzado en garantizar el aumento de cobertura y el ingreso de estudiantes nuevos [14].

Ahora bien, entrando en un análisis detallado del fenómeno de la deserción, se encontró que de los 24 departamentos sobre los cuales se dispone información, 12 (que equivalen al 50\%) presentan una tasa de deserción por cohorte superior a la media nacional; sin embargo, aquellos que están por debajo apenas alcanzan ese nivel, pues se encontró que solo 3, de los 24 departamentos, están por debajo del 40\% (MEN, 2013). Respecto a las disciplinas académicas, $[5,9,15]$ afirman que los índices más altos de deserción están ligados a las profesiones relacionadas con Ingeniería, Arquitectura, Urbanismo y afines; en las que se alcanzan niveles de deserción por cohorte superiores al 55\%, lo cual implica que menos de la mitad de los estudiantes logran obtener su título profesional.

La Universidad de la Amazonia es una institución de educación superior de carácter oficial, transformada por la Ley 60 de 1982, que tiene por mandato y objetivo atender al 35\% del territorio nacional, pues sobre sus hombros pesa la responsabilidad de brindar el servicio educativo en el nivel universitario en los departamentos de Amazonas, Guanía, Guaviare, Vaupés, Putumayo y Caquetá. No obstante, en las actuales condiciones financieras y administrativas, solo atiende al departamento del Caquetá, donde se encuentra su sede principal, y al departamento del Amazonas con la extensión de algunos programas por única cohorte.

Respecto al fenómeno de la deserción en la Universidad de la Amazonia, la Oficina Asesora de Planeación - OAP [16] afirma que la deserción anual siempre ha estado por debajo de la media nacional, sin embargo, no existe un registro detallado de deserción por cohortes de los diferentes programas ofertados por la institución, lo que genera dispersión al momento de caracterizar el fenómeno en dicha universidad. No obstante, la información suministrada por la OAP tiene armonía con los indicadores del Ministerio de Educación, pues Caquetá reporta en los últimos años una sensible disminución de la deserción [17].

Ahora bien, respecto al programa de Ingeniería de Sistemas, se encontró que, al igual que las tendencias nacionales, los indicadores de deserción por año superan el promedio institucional, pues oscilan en $13.4 \%$, mientras que el promedio nacional está en $11.98 \%$ y el institucional en $8.37 \%$ [16]. Este escenario genera un cuestionamiento institucional y es en relación a las causas que generan en el Programa de Ingeniería de Sistemas un nicho confortable para el fenómeno de la deserción y el significado de estas causas.

De esta manera, el presente manuscrito tiene como objetivo principal identificar 
las causas de la deserción en el Programa de Ingeniería de Sistemas y poder entender el sentido de dichas causas. Para ello el documento realiza inicialmente un riguroso análisis teórico de las diferentes corrientes alrededor del tema de la deserción y los conceptos que esta involucra. Luego identifica y caracteriza los desertores como población participante del estudio, para, a partir de herramientas cuantitativas, determinar tendencias de los posibles factores que agudizan la deserción y su expresión en términos numéricos.

Finalmente, se presenta una aproximación de análisis de corte más cualitativo que pone sobre la mesa la incertidumbre y las desconfianzas que solo un dato cuantitativo puede generar cuando se analiza solo sin adentrarse en las sensibilidades de los participantes. Se espera que este documento sea de utilidad para invitar a la reflexión de la forma en que hacemos educación, no solo en la relación docente-estudiante, sino en la relación padre-hijo y otras situaciones en contextos similares.

\section{Materiales y métodos Localización y caracterización de la población objeto del estudio}

El estudio se llevó a cabo en la Universidad de la Amazonia, localizada en el barrio Porvenir en el municipio de Florencia en el departamento del Caquetá. La población que participó en la presente investigación pertenece a los desertores del programa de Ingeniería de Sistemas de la Universidad de la Amazonia en los periodos académicos comprendidos entre 2012-I y 2015-I.

\section{Enfoque metodológico}

La investigación fue de tipo mixto, desarrollada a partir de técnicas cuantitativas y cualitativas, dentro de un enfoque descriptivo que permitió identificar aquellos asuntos que motivaron a los estudiantes de Ingeniería de Sistemas de los periodos 2012-1 y 2015-1 a desertar de la carrera.

\section{El método}

La identificación se hizo a través de sucesivos trabajos de consulta y rastreo en las diferentes bases de datos disponibles en la Plataforma Chaira de la Universidad de la Amazonia. Se identificaron los niveles de deserción que existían a nivel general y, posteriormente, se fue filtrando por facultades y, finalmente, al Programa de Ingeniería de Sistemas - PIG, lo cual fue contrastado por el SPADIES. Luego se filtró la información de acuerdo con el periodo seleccionado y se codificaron los ingresos y permanencias y de esta manera se identificaron los desertores.

Para el diseño del instrumento con el cual se haría el levantamiento de la información primaria, se realizaron algunos diálogos informales y la observación participante de algunos desertores y algunos estudiantes con el interés de desertar. A partir de ahí se identificaron algunas variables a tener en cuenta en el formulario versión cuestionario que diligenciarían los estudiantes. Se hizo la guía de diligenciamiento y se efectuaron algunas pruebas test y con sus resultados los respectivos ajustes.

Al ser el cuestionario una herramienta que no exige la presencia del orientador ni el encuestador, que posibilita una relación más cercana entre la pregunta y quien la

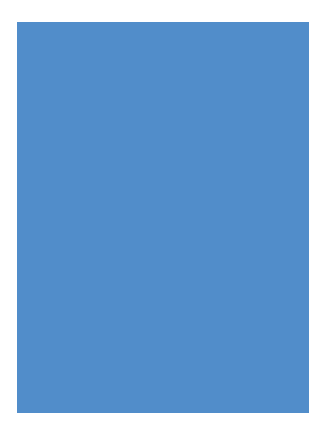


responde, se optó por colocar el formulario ajustado, de manera virtual a través de Google Drive. El formulario constó en su primera parte de 24 preguntas cerradas, las cuales fueron enviadas por correo electrónico a cada uno de los 289 desertores, con lo cual se buscó garantizar que llegara solamente a los individuos que realmente habían dejado sus estudios de Ingeniería de Sistemas. Posteriormente, se estableció comunicación vía telefónica con algunos que no habían respondido por el correo electrónico, para finalmente contar con 136 de ellos, es decir, el 47.5\% de los desertores diligenciaron el cuestionario. Los datos fueron llevados a una base de datos en el programa Excel y se realizaron los respectivos análisis numéricos.

En la segunda parte del cuestionario, se consignaron preguntas de tipo cualitativas, cuyas variables de análisis correspondieron a la opinión sobre las causas que lo motivaron a desertar y el imaginario de ser profesionales. Una vez realizada la encuesta, se identificaron tendencias y se eligieron al azar 10 desertores con los cuales se realizaron diálogos informales, los cuales fueron categorizados y triangulados con la información cuantitativa.

\section{Resultados y Discusión Identificación de los desertores}

Díaz [18] define la deserción estudiantil como el abandono voluntario del sistema educativo, el cual puede ser leído desde diferentes aristas como las socioeconómicas, personales, institucionales y académicas. Igualmente, afirma que la forma como estas se interpreten dependerá de quien las lea, es decir, bajo las gafas de la individualidad, la institucionalidad o la administración pública.

En este primer momento, se muestran los resultados de manera institucional, es decir, la cantidad de estudiantes que desertaron en cada uno de los periodos académicos y su respectiva representación porcentual respecto al total de los matriculados para el mismo periodo de referencia. La investigación arrojó que en el I-2012 desertaron 26 estudiantes, los cuales representaban el 9\% del programa; durante el II-2012 desertaron 40 estudiantes que representaban el 14\% de los matriculados en ese periodo; en el I-2013 se reportó una tasa de deserción del 11\%, equivalente a 32 estudiantes; en el segundo periodo académico del 2013 se registraron 46 desertores, equivalentes al 16\% de la población estudiantil; en el I-2014, el abandono académico representó el 17\% con 49 estudiantes; en el II-2014 se registraron 37 casos, equivalentes al 13\%; finalmente, para el primer periodo del 2015 se reportaron 59 casos, equivalentes al 20\% de la población matriculada.

Entre el periodo comprendido entre los años 2012 y 2015, se identificaron 289 estudiantes desertores, de los cuales 266, es decir el $92 \%$, se retiraron definitivamente y solo el 8\%, que equivale a 23 individuos, volvieron a seguir estudiando.

Llama la atención que los indicadores de deserción semestral se hayan sostenido e incluso en algunos casos aumentado luego del I-2014, periodo en el que la Universidad de la Amazonia, a través de un convenio con el Departamento para la Prosperidad Social - DPS, implementó el programa Jóvenes en Acción, el cual tiene como uno de sus principios rectores aportar a la disminución de la deserción estudiantil a través de las transferencias 
monetarias condicionadas - TMC [19].

\section{Caracterización de los desertores}

El primer abordaje de la caracterización fue el sexo. De esta manera se encontró que, de los 136 estudiantes desertores consultados, 123 eran hombres, quienes representan el $90 \%$, y solo 13 mujeres, quienes representan el 10\% de la muestra. Este escenario es contrario al expuesto por Jaramillo [20], quien expone que existe poca diferencia en las proporciones de hombres y mujeres con estudios universitarios incompletos, desvirtuando al sexo como factor determinante en el abandono o postergación de los estudios universitarios. El presente caso de estudio muestra una clara incidencia del género en la deserción universitaria al interior del programa de Ingeniería de Sistemas de la Universidad de la Amazonia.

Todo apunta a que este caso de estudio tiene mayor relación en lo planteado por el Centro de Estudios sobre Desarrollo Económico - CEDE [21], quienes sostienen que la deserción afecta con mayor fuerza a los hombres que a las mujeres, con diferencias que en ocasiones alcanzan los 10 puntos porcentuales. En el mismo sentido, Montes et al. [22] sostienen que los hombres son mucho más propensos a desertar, pues, en el caso de la Universidad Eafit, casi el $60 \%$ de las personas que abandonan los procesos de formación profesional son hombres. Este planteamiento es secundado por Malagón et al. [23], quien afirma que los hombres tienen mucha más probabilidad de desertar que las mujeres, pues sostiene que estas poseen un mayor sentido de responsabilidad y perseverancia.

Finalmente, el MEN [24], al analizar la deserción por cohortes en la educación colombiana, considera pertinente concluir que los hombres tienen una mayor tasa de deserción que las mujeres, la cual está sobre el $50.9 \%$, mientras que la de las mujeres alcanza un $43 \%$. Este escenario deja en evidencia la incidencia del género en el fenómeno de deserción, situación, que tal y como lo plantea Cabrera et al. [25], deberá ser objeto de reflexión social e institucional al interior de la universidad.

En relación a la edad, la investigación arrojó que la mayoría de estudiantes en condición de deserción se encuentran en el rango entre 20 y 25 años, lo cual localiza al fenómeno en la población estudiantil relativamente joven. Sobre el particular, Meléndez \& Meriño [26] expresan que, en el caso de la Facultad de Ingeniería de la Universidad de la Guajira, los jóvenes con edades entre 20 y 24 años son la población con mayor susceptibilidad. En el mismo sentido, el CEDE [21] sostiene que el rango de edad que presenta mayor porcentaje de desertores está entre los 21 y 25 años. Malagón et al. [23] sostienen que la población con mayor grado de vulnerabilidad a la deserción se encuentra en el rango de 22 a 24 años.

La Universidad Nacional [27] sostiene que las personas con 19 años o más presentan mayor vulnerabilidad ante el fenómeno de la deserción, situación que motivan en mayores responsabilidades económicas y familiares. El mencionado escenario posee menor impacto para quienes ingresan sobre los 16 años a la universidad. En el mismo sentido, García (1987), citado por UAPA [28], afirma que cuanto más joven se ingresa al sistema universitario, mayor probabilidad de éxito se tiene, no obstante, en sus estudios no encuentra relación con el rendimiento académico. Finalmente, el 
MEN [15] sostiene que, a nivel nacional, los estudiantes que ingresan al sistema universitario a una mayor edad pueden acumular tasas de deserción por cohorte hasta $17 \%$ más altas. Igualmente, afirma que dicho comportamiento puedo obedecer a variables laborales, económicas y familiares.

En términos del lugar de procedencia, se evidenció que de los 136 encuestados, el grueso de la población desertora pertenece al departamento del Caquetá en un $92 \%$, el $8 \%$ restante procede del departamento del Huila. Los municipios con mayor participación son Florencia con un 38\%; El Paujil con 9\%; El Doncello y Milán con 7\%; Curillo, Belén, Morelia (Caquetá) y Pitalito (Huila) con 6\% cada uno; el restante $15 \%$ está representado por Cartagena del Chairá, La Montañita, Puerto Rico y San Vicente (Caquetá) y Neiva (Huila).

El anterior escenario permite evidenciar que la ubicación de la universidad tiene estrecha relación con el lugar de procedencia de los desertores, pues, si se hace una lectura desde municipios, se encuentra que el 38\% están relacionados con la ubicación del campus principal de la universidad; si se hace una reflexión por departamentos, el porcentaje es mucho más generoso pues asciende al 92\%. Estas realidades son muy similares a las encontradas por algunos autores, quienes, al realizar un análisis sobre la deserción en la Universidad Javeriana de Bogotá, encontraron que el 77.2\% de los desertores eran oriundos de la capital y el restante $22.28 \%$ procedían de ciudades como Bucaramanga, Cartagena y Cali. En el mismo sentido, Patiño \& Reyes [29], al realizar un análisis de la deserción en la Universidad de Nariño, sostienen que el 80.76\% de los desertores eran procedentes de Pasto.
El siguiente factor de análisis es la formación académica, la cual hace referencia a la formación académica de la básica y la media. Según la UAPA [28], para el año 2004 de cada 100 estudiantes que ingresaron a la escuela, solo 47 lograron terminar bachillerato y, de estos, solo 27 ingresaron a la educación superior. Además del escaso número de quienes ingresaron, solo un poco más de la mitad terminaron exitosamente sus estudios y se titularon en cualquiera de las modalidades de educación superior (técnica, tecnológica o universitaria). El anterior escenario reviste de validez lo planteado por Cabrera et al. [25], quienes afirman que dentro de las causas más significativas para la deserción en el sistema universitario se encuentran las evolutivas, institucionales, psicoacadémicas, entre otras.

Ahora bien, al analizar los datos arrojados por la investigación se encontró que de los 136 estudiantes desertores, el 32\% proceden de modalidad académica; $27 \%$ culminaron sus estudios de bachiller en modalidad de Computación/Informática; procedentes de modalidad Comercial/ Administrativa registra el $24 \%$ de la población; 10\% procedente de modalidad Agropecuaria/Ambiental; y el restante 7\% tuvieron como modalidad Dibujo, Pedagogía, entre otros. Al analizar el tipo de institución de la cual fueron egresados, se encontró que en un 95\% proceden de colegios públicos.

Hernández-Mata et al. [30] exponen que el lugar de procedencia académica (tipo de colegio del que egresan de bachillerato) es el principal factor de riesgo para la deserción, situación que justifican en los diferentes niveles de exigencia y disciplina impartidos por las instituciones de formación básica, así como la relación o no con 
la disciplina elegida. No obstante, para nuestro caso de estudio es complejo realizar una disertación en torno al tema, pues en el Departamento del Caquetá existen 151 establecimientos de orden público y tan solo 3 privados con el ciclo de bachillerato completo [31].

Por otro lado, al indagar sobre la percepción alrededor de la formación básica y secundaria como factor influyente en la deserción académica, se encontró que el 25\% está totalmente de acuerdo en que ese factor influyó en su deserción: $40 \%$ de los encuestados respondió estar de acuerdo; 30\% respondió estar parcialmente de acuerdo; mientras que solo el 5\% restante considera que no existe correlación alguna. Patiño \& Reyes [29] exponen que uno de los factores relacionados con la deserción está relacionado con la formación del bachillerato, pues su investigación detectó falencias en los procesos de formación media, situación que incide negativamente en el transcurrir académico de la universidad. Igualmente, Bravo \& Mejía [5] exponen que las menores deserciones corresponden a estudiantes de estratos altos, quienes a la vez tienen la mayor probabilidad de obtener mejores resultados en su bachillerato.

Finalmente, Isaza, citado por Olave-Arias et al. [32], expone que las causas académicas son determinantes al analizar el fenómeno de la deserción, pues se encontró que entre los estudiantes que ingresan con mejor puntaje del Icfes, y los de menor, la diferencia en el grado de deserción alcanza el 20\%, situación que está en total sintonía con lo planteado por el MEN [24], que sostiene que los estudiantes que ingresaron con clasificación baja en las pruebas Saber 11 presentan una tasa de deserción por cohorte del 55\%, mientras que para los clasificados en Alta esta asciende a $35 \%$.

\section{Ingresos y ayudas económicas a los desertores}

Las estadísticas encontradas por la Universidad de Chile (2008) establecen que las causas con mayor incidencia en el primer año universitario están relacionadas con problemas vocacionales, rendimiento académico y situación económica familiar. Para Elias \& Moreno, citados por Martínez et al. [33], las razones de deserción académica en torno a los problemas económicos están relacionadas con la falta de recursos en el hogar para hacer frente a los gastos que demanda la escuela, el abandono para trabajar o buscar empleo. En el mismo sentido, Sánchez et al. (2009) establecen que la ausencia de recursos durante el proceso de formación genera desmotivación y falta de compromiso con sus responsabilidades académicas, lo que impide la realización de su proyecto de vida como profesional.

Barrios [34] expone que los estudiantes provenientes de hogares con mayor flexibilidad económica tienen menor probabilidad de deserción que aquellos provenientes de hogares con niveles de ingresos más bajos. Para Espinoza et al. [35], la deserción por factores económicos tiene raíces un poco más profundas en los estudiantes, dentro de las cuales se encuentra el poco valor que se le atribuye a la formación académica y la generación de recursos en el corto plazo.

Ahora bien, para nuestro caso de estudio, al indagar sobre la procedencia de los ingresos al momento del retiro de la universidad, se encontró que el 38\% dependían totalmente de sus ingresos familiares; el 
33\% sostuvo que sus ingresos provenían de sus padres, pero que paralelamente a la universidad desarrollaban algún tipo de actividad laboral que les generaba recursos adicionales; el 28\% respondió ser totalmente independientes y por ende responsables de su sustento; finalmente, el 1\% sostuvo que poseía una beca para el desarrollo de su formación profesional. Igualmente, se indagó sobre el estrato socioeconómico. Se encontró que el 74\% pertenecían al estrato 1 , y el restante $26 \%$ al estrato 2. Esta última condición, según Londoño [36], es crítica para la deserción, pues afirma que pertenecer a los estratos 1 y 2 representa más del 70\% de las causas relacionadas con la deserción por situaciones económicas.

Al indagar sobre la actual condición laboral de los desertores, se encontró que el $70 \%$ están vinculados laboralmente, situación que, al contrastarla con su procedencia socioeconómica, le otorga razón a los planteamientos teóricos expuestos con anterioridad, pues la gran mayoría proceden de hogares con situaciones económicas limitadas y cambiaron su rol de estudiante universitario por el de trabajador. En palabras de Román [37], la pobreza y los bajos ingresos son claros determinantes de la deserción, especialmente porque estos contextos inducen a la búsqueda de trabajo y ponen en cuestionamiento los costos de oportunidad real para continuar con los procesos de formación en función del beneficio futuro.

\section{Percepción de los desertores}

Alcázar [38] afirma que los beneficios generados por los procesos educativos en el estudiante dependen en gran medida de la calidad de la educación impartida durante el proceso de formación. Igualmen- te, sostiene que la deserción puede estar asociada a la valoración individual de la educación, por lo cual, la calidad del sistema educativo y su poca armonización con las necesidades de los jóvenes son factores influyentes para la deserción. En el mismo sentido, Duque \& Chaparro [39] sostienen que la incidencia de la educación en el desarrollo de las comunidades es algo innegable, por lo que generar procesos educativos de calidad para el estudiante, máxime en nuestros actuales tiempos, es indispensable para mantenerlos en el sistema educativo.

Al indagar sobre la percepción de los desertores acerca de la calidad académica del programa de Ingeniería de Sistemas, se encontró que el 17\% de los encuestados clasificó la calidad de la educación como excelente; el 60\% considera que la calidad impartida es buena; 19\% consideran que es regular; finalmente, el restante $4 \%$ considera que es mala. Este escenario permite evidenciar que la calidad del servicio educativo no es una variable asociada a la deserción, pues el 77\% de los encuestados clasificaron el servicio educativo como excelente y bueno. En palabras de Sánchez et al. [40], los esfuerzos institucionales por generar procesos de calidad en las universidades, especialmente en las públicas, son verdaderos esfuerzos titánicos, dada las actuales condiciones económicas y financieras de las mismas.

Por otro lado, respecto al tema de los educadores, Segura [41] expone que la labor docente tiene una gran incidencia sobre la formación académica y el desarrollo del estudiante, pues considera el ejercicio docente como un verdadero compromiso social. Desde otra arista, Melo et al. [42] sostienen que las dificultades en la contratación docente y las pocas oportunida- 
des para la formación doctoral configuran afectaciones sobre las actividades de investigación, la formación académica de los estudiantes y en general los resultados del sistema educativo, situación que puede incidir sobre los estudiantes y la percepción que configuren en torno al cuerpo docente y la institución.

Para el presente caso de estudio, al auscultar sobre las competencias disciplinares del personal docente vinculado al programa, se evidenció que el imaginario de los desertores sobre las competencias profesionales de los docente es positiva, por lo que se descarta relación alguna con el fenómeno de deserción, al menos de forma directa, pues el 10\% de los encuestados clasifica a los profesores en un nivel muy alto; $48 \%$ de los encuestados considera que tienen un nivel alto; $36 \%$ considera que son buenos profesionales; $6 \%$ considera que su nivel de competencia profesional es regular; ningún estudiante conceptuó de manera negativa.

En lo referente a la condición humana del docente, Guevara [43] afirma que una de las características esenciales de este como ser humano debe ser despertar pasión intelectual, fomentar el desarrollo de condiciones humanas en los estudiantes, generando confianza en sí mismos y, por tanto, desarrollo integral. Al indagar acerca de la percepción de los desertores en torno a las competencias humanas del personal docente, se encontró que el 16\% de los encuestados considera que poseen excelentes competencias humanas; $60 \%$ las clasificó como muy buenas; $18 \%$ considera que son buenas; el restante $6 \%$ considera que son regulares; al igual que en las condiciones académico-profesionales, ningún estudiante opino de manera negativa.
Ahora bien, en los procesos de formación académica, al igual que en muchos otros, la pasión y convicción por lo que se hace genera altos grados de satisfacción, los cuales se convertirán en una fuerza poderosa de motivación para estudiante, pues su contexto educativo se convierte en un recorrido hacia su meta de profesionalización, situación que convierte a la universidad en un verdadero centro de desarrollo integral. De Vries [44] expone que existen cuatro tipos de desertores: quienes abandonan por motivos personales, quienes pierden varios espacios académicos y deciden desertar, quienes tienes horarios y dificultades para asistir a la universidad y, finalmente, quienes abandonan la carrera por vocación. Estos últimos abandonan sus estudios por no sentirse satisfechos con los procesos académicos y por considerar que no era su vocación.

Herrera [45] propone que la escuela debe considerar como factor endógeno de constante revisión a la calidad pedagógica, pues su desatención genera desvalorización de la escuela y el retiro de los alumnos del sistema educativo. Sobre el nivel de satisfacción académica, se encontró que el 20\% lo consideran alto; 59\% afirman estar en rango medio; finalmente, $21 \%$ expresa niveles de satisfacción bajos. Igualmente, se encontró que el 55\% de los encuestados consideran que el alto nivel de exigencia académica fue determinante en su deserción, mientras que el restante $45 \%$ no considera que exista vínculo alguno.

Estos escenarios tienen estrecha relación con la intencionalidad de reingreso de los encuestados, pues el 51\% considera procedente retomar sus estudios. Estos estudiantes muy seguramente pertenezcan a estudiantes satisfechos sin incidencia de la exigencia académica como factor de de- 
serción, es decir, personas que pese a su condición de deserción tienen vocación hacia la Ingeniería de Sistemas. El 28\% que respondió que no está interesado en retomar sus estudios, muy seguramente configura un estudiante afectado por los niveles de exigencia académica y con bajos niveles de satisfacción durante su estadía. Finalmente, el 21\% que presenta indecisión por reingresar pueden constituir el grupo satisfacción media/baja con afectación académica.

Finalmente, se indagó sobre el horario más conveniente para quienes desean o aspiran reingresar al programa. Se encontró que de los 70 estudiantes interesados en regresar, el 50\% afirma que la oferta del programa en jornada nocturna facilitaría su ingreso; la jornada diurna y fines de semana comparten posición con $21 \%$ cada una; solamente el $8 \%$ contempla una metodología virtual. En palabras de Román [46], estos ajustes a las políticas educativas y al sistema escolar son necesarios para el ofrecimiento de programas con calidad total, que generen satisfacción en los estudiantes.

\section{Una aproximación antropológica a un problema educativo}

Al realizar un análisis más de corte cualitativo, al conversar con los desertores, fue posible reforzar algunos planteamientos hechos a la luz de los datos obtenidos a través de las encuestas y repensar algunos. Desde la arena antropológica, Arana \& Bianculli mencionan que desertar de un proceso educativo corresponde a abandonar los ideales o las obligaciones que guiaron una decisión de vida en un momento de elección. Precisan que el abandono puede ser generado por distintos motivos personales, familiares, econó- micos o sociales. De igual forma, agregan las autoras, siguiendo a García de Fanelli, que estos factores pueden ser endógenos o exógenos a la institución. En los exógenos están el género, edad, el lugar de residencia, el nivel socioeconómico, el nivel educativo de los padres, las aspiraciones y motivaciones personales y las propias actitudes; y en los endógenos se identifica la existencia de políticas de admisión, la relación con la formación previa del estudiante, la calidad del cuerpo docente, el equipamiento y la duración del plan de estudios. Magrassi \& Roca plantean que el individuo como persona aparece como microcosmos de la fisonomía grupal de su cultura. ¿Qué es lo que hay en la cultura, en el imaginario de vida de nuestros jóvenes caqueteños, que hace que el fenómeno de la deserción crezca constantemente? Durante las conversaciones con los desertores, al abordar los aspectos causales de mayor importancia de su deserción, sus expresiones estuvieron reiterativamente relacionadas con situaciones como: "dificultades económicas", "bajo rendimiento académico" y "me quería cambiar de carrera".

El primer factor mencionado, que corresponde a las "dificultades económicas", no sorprende puesto que diferentes autores lo han señalado con bastante frecuencia, sin embargo, en este caso algunas situaciones podrían resultar sospechosas y hasta contradictorias a la hora de caracterizar el contexto del factor. En la cotidianidad es usual escuchar comentarios ligeros como: "ando en este carro porque no hay dinero para comprar otro mejor", "estudié aquí porque no había dinero para ir a otra ciudad", "la casa está que se cae porque no hay dinero para arreglarla", "los pobres son pobres por falta de dinero". Parece, entonces, que la pobreza está asociada 
con una falta notable de dinero y que la ausencia de este es la causa directa de que muchas cosas no se realicen, incluso la causante de no poder seguir estudiando. Gómez y colaboradores, en un reciente estudio realizado en la Universidad de la Amazonia con estudiantes beneficiarios del Programa Jóvenes en Acción de la Presidencia de la República, mediante el cual se da subsidio a de 2.233 estudiantes en esta alma mater desde el año 2014 a través de las llamadas Transferencias Monetarias Condicionadas - TMC; encontraron que el 95\% de los estudiantes beneficiarios del programa ven las TMC como un complemento económico para desarrollar su proceso educativo, pero admitieron que podrían desarrollar su estudios tanto con ellas como sin ellas . Así, al observar el periodo de estudio desde que inició el programa, la tasa de deserción sigue incrementándose [47].

Por otra parte, se tiene que los valores de las matrículas de los estudiantes de pregrado en la Uniamazonía se guían por una tabla única de matrícula, de modo que el valor de la matrícula por semestre depende de los ingresos económicos reportados por el mismo estudiante; así las cosas, la diferencia del costo del semestre entre carreras es poco significativo. Ahora, los desertores expresan que se retiraron por motivos económicos, pero al conversar acerca de sus pensamientos antes de tomar la decisión de retirarse definitivamente de la carrera, estos estuvieron relacionados en un $25 \%$ con "pensé en cambiar de carrera", un 19\% con "pensé en cancelar una o más asignaturas", un 14\% de ellos "se cambió de una vez de carrera" y el 24\% "cancelo semestre". Si el desertor antes de desertar contempló opciones como estas, la motivación o causa de la deserción no fue, obviamente, las "dificultades económicas", teniendo en cuenta que las diferencias en los valores de la matrícula son insignificantes y no se pagan por número de asignaturas vistas. En total, estaríamos hablando del 76\% de los desertores en esta condición.

De igual forma, si de repente el tema de colegiaturas estuviera solucionado y a lo que se refieren los desertores con "dificultad económica" está más relacionado con el pago de manutención, resulta contradictorio entonces que los que más desertaron fueron los estudiantes que tienen su origen en la ciudad de Florencia, donde tienen su hogar y obviamente las condiciones de alojamiento y manutención podrían ser menos complejas de lograr. Aunado a lo anterior, es preciso mencionar que existen herramientas que podrían ayudar a mitigar las dificultades económicas, como por ejemplo los subsidios alimentarios, los almuerzos que entrega la Universidad a través de Bienestar universitario, los beneficios por el Programa Jóvenes en Acción y los créditos ICETEX para el pago de sus colegiaturas. Es por todo lo anterior que se considera que este factor, aunque es mencionado por los desertores, no sería el detonante para su decisión de abandonar sus estudios, o por lo menos no en la mayoría de los casos.

Respecto al segundo factor mencionado, que fue el "bajo rendimiento académico", aunque los desertores resaltan la buena calidad académica del programa, de los docentes y de los equipos; consideran que la formación básica primaria y secundaria es determinante para tener buen rendimiento académico en la universidad y que sentían que les había faltado una buena preparación en la básica. Esta percepción estudiantil coincide con lo propuesto por el CEDE [21], que enfatiza que uno de 
los determinantes para la deserción estudiantil es lo académico, relacionado con el tipo de colegio donde el estudiante hizo la secundaria. Si el énfasis del colegio donde el estudiante terminó su secundaria no tenía nada relacionado con el perfil de la carrera escogida, va a ser más complejo el aprendizaje y el rendimiento.

El otro factor mencionado por los desertores fue "me quería cambiar de carrera", contradictorio con el primer factor "dificultades económicas". Semióticamente expresa insatisfacción, desilusión, disgusto y desinterés por continuar en aquello que eligió. La situación se torna más delicada al revisar la información cuantitativa, pues compartieron que al ingresar al Programa Ingeniería de Sistemas estaban indecisos sobre lo que querían estudiar (39\%); que no tenían ninguna idea de que estudiar (11\%); más grave aún, los restantes confesaron no tener ganas de realizar estudios universitarios (3\%). Es decir, solo el $47 \%$ dijo estar seguro de que quería estudiar esa carrera al inicio, pero al realizar las entrevistas una de las categorías más frecuentes fue "me quería cambiar de carrera".

En resumen, dejando de lado el tema económico que ya se discutió, se tiene que un poco menos de la mitad de los desertores no tenían claridad sobre lo que querían estudiar, se querían cambiar de carrera o sencillamente no querían estudiar. Este grupo de desertores se denominará en adelante y solo para efectos de este análisis "los obligados". En el otro grupo están los que sí estaban felices y querían estudiar esta carrera. Para efectos de la explicación se denominarán "los identificados".

Finlandia es uno de los países con los mejores resultados en educación y con meno- res índices de deserción escolar, pues a la par de tener resuelto el tema de cobertura, la calidad si bien se basa en el cumplimiento de los estándares internacionales en términos de conocimiento, hay una marcada preocupación por velar por las capacidades, las aptitudes y la cultura de toda la población estudiantil, al ser el principio de la educación permanente ofrecer la posibilidad de aprender algo nuevo durante toda la vida. En este tipo de educación existe una marcada preocupación por el ser y su desarrollo, por lograr que el estudiante tome sentido e interiorice la importancia de estudiar. Esto solo lo han logrado a través de formar a los estudiantes con conocimientos y destrezas que sean útiles para su vida. Para ello se trabaja insistentemente en una amplia diversidad de asuntos que permiten que el estudiante se identifique con alguna disciplina en particular, además de temas bastante sensibles para su futuro independiente como, por ejemplo, la economía doméstica [48].

Al finalizar la escuela primaria los jóvenes finlandeses se encuentran frente a una importante elección: continuar desarrollando su cultura general en el bachillerato o comenzar una formación profesional en un instituto. Con ello se busca que el estudiante tenga la madurez suficiente al momento de elegir lo que guiará su vida profesional [48].

La situación es un poco diferente en Colombia, pues existe un marcado esfuerzo de las comunidades integrantes de las políticas de educación, esfuerzos que están destinados a cumplir con el incremento de las tazas de cobertura media anual y a posicionar al país en altos estándares de calidad, esto tanto a nivel de educación superior como desde la básica y media [49]. Dicho sea de paso, el país tiene como 
metas del milenio en educación tener educación secundaria universal, que todos los niños de 7 a 17 años estén en una institución escolar y cursen el grado que corresponde a su edad y bajar la tasa de analfabetismo a cero [50].

En estas metas y énfasis ¿dónde queda el ser humano que es el estudiante, más allá del cúmulo de conocimientos?, ¿dónde está el espacio para la reflexión de los jóvenes, para que identifiquen el qué me gusta, si no conocen y practican diferentes opciones para identificar sus habilidades y comodidades, cómo darán con el qué me apasiona?, ¿dónde está la preocupación por velar por las capacidades, las aptitudes y la cultura de este estudiante? Es preciso mencionar que en las amplias conversaciones realizadas con los desertores, estos coincidieron en que nunca tuvieron la posibilidad de elegir el énfasis del colegio en el que iban a estudiar. La decisión fue más obligada que motivada, en algunos casos porque era lo único que se ofrecía en el colegio del pueblo de donde son originarios. En el caso de los desertores originarios de Florencia, esta decisión estuvo relacionada con aquel lugar donde lograron un cupo. Una vez dentro de este esquema de formación, no tuvieron momentos que permitieran la posibilidad de explorar otros énfasis que les permitieran aproximarse a algún tipo de conocimiento, actividad o destreza que los llenara o los apasionara: arte, pintura, música, agropecuario, electrónico, comercial, técnico o arquitectónico. Así las cosas, el asunto para ellos fue cumplir con la formación secundaria, pero no se lograron enamorar de algo en particular a partir de esta formación, ¿cómo puedes elegir algo para tu proyecto de vida, si no lo conoces al menos un poco?, máxime en un tiempo de cambios instantáneos, en el que no hay tiempo para pensar, menos en el futuro.

Difícilmente un desertor, en su calidad de estudiante "obligado", va a estar abierto a buscar soluciones y empeño para lograr desarrollar un proceso del cual no está convencido y no está convencido porque aunque fue su decisión no tenía un plan de vida alrededor de esta elección, en la mayoría de los casos no hubo un antecedente para hacerlo llegar a este mundo disciplinar que eligió.

De Vries [44] expone que existen cuatro tipos de desertores: quienes abandonan por motivos personales, quienes pierden varios espacios académicos y deciden desertar, quienes tienen horarios y dificultades para asistir a la universidad y, finalmente, quienes abandonan la carrera por vocación. Estos últimos abandonan sus estudios por no sentirse satisfechos con los procesos académicos y por considerar que la carrera no era su vocación. Sin embargo, la situación del vaivén de la mala elección de la carrera de acuerdo a su gusto se confunde con la nula planeación de vida del joven y la no vocación o ilusión por desarrollar una formación profesional, el no interés por estudiar. En línea con Román [37], los factores culturales, el universo de las significaciones que enmarcan sus vidas cotidianas y la trama de la decisión de los sujetos sociales. La actitud, la expectativa hacia la educación, la valoración y los consumos culturales que los jóvenes tienen, serían fuertes detonantes detrás de un proceso de deserción.

Ahora, al observar la cotidianidad de los desertores que aseguraron haber estado seguros de su carrera y conversar acerca de si les gustaría regresar, solo la mitad dijo que sí, los demás dijeron que no o estar indecisos, es decir, existe una decisión 
que le antecede a todo esto y está relacionada con qué tanto se desea ser profesional, independiente de que no se tenga claro aún en qué carrera.

De esta manera, tanto a este grupo de los "identificados" como al de los "obligados" los acompaña un factor que es exógeno a la institución, que tiene que ver con los determinantes sociales y culturales que son más de contexto y constituyen un importante conjunto de variables que refieren a sus modos de interpretar la realidad y a las dinámicas y tipo de relaciones en el ámbito familiar, comunitario, social y político.

El agotamiento del modelo modernizador, la crisis crucial que afecta a la familia y las fallas de las instituciones políticas y educacionales colocan a los jóvenes colombianos en una situación de aislamiento. El temor de un futuro de desempleo o subempleo los embarga y los limita a dar un paso para regresar a las aulas [51], pues consideran que se puede sobrevivir en el informalismo, lo que, en algunas ocasiones, suena más atractivo que ser un profesional desempleado.

Infortunadamente, la educación no es entusiasta y no asegura ni empleo ni movilidad social, en una sociedad sin un modelo claro de futuro en el cual ellos puedan insertarse. Máxime en un lugar como Florencia y el Caquetá, limitado de oportunidades y en donde las que se dan han estado mediadas, más que por una formación profesional, por etapas de boom, por ejemplo: del caucho, de la coca, de las pirámides, del paramilitarismo, donde tener acceso al dinero es más importante que tener educación.

Además, la ciudad es una capital que al- berga un número considerable de desplazados por la violencia que ahora viven paternalmente del Estado, los adolescentes están creciendo en medio de esta situación y es el referente de vida que están teniendo. La juventud se enfrenta entonces a un caos de valores, generado por lo que desde hace unos años Sandoval [51] llamó una sucesión muy rápida de tres situaciones sociales: la sociedad rural, la sociedad moderna y la sociedad sin modelo; en este caso más una sociedad sin modelo, la consolidación de formas organizadas del comercio informal, la economía subterránea, la organización económica de la droga o la dependencia de ella, la corrupción en el mundo financiero y administrativo y las visiones consumistas presentadas por los medios de comunicación de masas. 


\section{Conclusiones}

os factores de deserción van mucho más allá de lo que alcanzan a perIcibir los jóvenes o de lo que arrojan los números producto de reiteradas consultas, entre ellos la falta de dinero. La deserción está más relacionada en el fondo con el poco interés en acceder a una formación profesional, la desconfianza en si estudiar vale la pena para tener una vida digna y las pocas claridades de lo que como ser humano se quiere y se espera de la vida, en la que el individuo está inserto y es el artífice de lo que suceda.

Indudablemente, existe poco esfuerzo en el trabajo de la básica y media para que los estudiantes exploren posibilidades que los motiven, pues con frecuencia los adolescentes que no terminan ni este ciclo expresan: "no serví para el estudio", como si viera el estudio como un asunto obligado para el que no sirvió. Siempre habrá algo que a un ser humano se le fa- cilite más, algo por lo que se apasione y pueda aportar a la sociedad. La clave para los educandos será entonces ayudarlos a esta exploración e identificación preparatoria, como si los prepararan para un Icfes o unas pruebas Saber, pensando como los finlandeses, en prepararlos para la vida.

Los enfoques de investigación en educación deben ser cada día más interdisciplinarios, desde un enfoque cuantitativo el número habla de un resultado concreto, desde un enfoque cualitativo se busca entender el porqué de ese número o qué se esconde detrás de este resultado, de esta manera se obtendrán análisis que realmente ayuden a comprender esta sociedad contemporánea. 


\section{Referencias Bibliográficas}

1. V. Tinto, "Definir la deserción: una cuestión de perspectiva", Revista de la Educación Superior, vol. 18, 1989.

2. P. Buentello, "Deserción escolar, factores que determinan el abandono de la carrera profesional, estrategias y condiciones para el desarrollo del estudiante", 2013.

3. R. Silva, "Deserción: ¿Competitividad o Gestión?", Revista Lasallista de Investigación, 2006.

4. O. C. Lopera, "Determinantes de la deserción universitaria en la facultad de economía de la Universidad del Rosario", Revista Borradores de Investigación, $\mathrm{n}^{\circ}$. 95, 2008.

70 5. C.M. Bravo y G. A. Mejía, "Los retos de la educación superior en Colombia: una reflexión sobre el fenómeno de la deserción universitaria", Revista de Educación en Ingeniería, vol. 5, 2010.

6. Ministerio de Educación Nacional MEN, Deserción estudiantil en la educación superior Colombiana: metodología de seguimiento, diagnóstico y elementos para su prevención. Imprenta Nacional de Colombia. Bogotá (Colombia), 2009.

7. T. Marina, "El abandono escolar", Avances en Supervisión Educativa, n. 14, 2011.

8. M.D. Silva, El abandono escolar como problema social: presencia, ausencia e involucramiento del mundo adulto en la construcción de comunidades de aprendizaje. Tercera Conferencia Latinoamericana sobre el Abandono en la Educación Superior (CLABES). Ciudad de México (México), 2013.

9. V. A. Roa, La preocupante situación de la deserción universitaria. Universidad del Norte, Barranquilla (Colombia), 2014. 10. M. Rojas, (2009). “El abandono de los estudios: deserción y decepción de la juventud", Revista Académica de la Facultad de Ciencias Sociales, vol. 4, 2009.

11. Comisión Económica para América Latina - CEPAL. Panorama Social en América Latina 2001-2002: Elevadas tasas de deserción escolar en América Latina, 2002. Disponible en: http://www.cepal. org/cgi-bin/getProd.asp?xml=/prensa/ noticias/comunicados/0/11260/P11 260. $\mathrm{xml}$

12. E. A. Salcedo, “Deserción Universitaria en Colombia", Revista Academia y Virtualidad, vol. 3, 2010.

13. Revista Dinero. (2009). Se mantiene la deserción universitaria. Disponible en:http://www.dinero.com/pais/ articulo/se-mantiene-desercion-universitaria/73933, consultado el 08.02.2016

14. Ministerio de Educación Nacional - MEN, Deserción estudiantil en la educación superior colombiana, 2008. Disponible en: http://www.mineducacion. gov.co/sistemasdeinformacion/1735/articles-254702_archivo_pdf_politicas_estadisticas.pdfmi

15. Ministerio de Educación Nacional MEN, Educación Superior. Boletín informativo número 14, 2010. Disponible en: http://www.mineducacion.gov.co/1621/ articles-92779_archivo_pdf_Boletin14.pdf. 16. Oficina Asesora de Planeación - OAP. Histórico de Deserción Estudiantil en la Universidad de la Amazonia. Universidad de la Amazonia, Florencia (Caquetá), 2015.

17. Sistema de Información para la Prevención de la Deserción en Educación Superior - SPADIES, 2015. Disponible: www. mineducacion.gov.co

18. P.C. Díaz, "Modelo conceptual para la deserción estudiantil universitaria chilena", Estudios Pedagógicos, vol. 34, 2008.

19. C.A. Gómez, C. V. Sánchez., y Y.H. Ri- 
vera, “Programa Jóvenes en acción en la Universidad de la Amazonia: una lectura desde los actores en cuestión", Revista Cooperativismo y Desarrollo, vol. 24, 2016. 20. V.M. Jaramillo, Estudio sobre la deserción y repitencia en la Educación Superior en Panamá. Ciudad de Panamá (Panamá), 2005.

21. Centro de Estudios sobre Desarrollo Económico - CEDE. Determinantes de la Deserción. Facultad de Economía, Universidad de los Andes. Bogotá (Colombia), 2014.

22. I. C. Montes, P. M. Almonacid, C. S. Gómez, F.I Zuluaga, Z. E. Tamayo, "Análisis de la deserción estudiantil en los programa de pregrado de la Universidad Eafit", Cuadernos de Investigación, 2010.

23. L.M. Malagón, C.A Calderón., y E.L. Soto, Estudio de la deserción estudiantil de los programas de pregrado de la Universidad de los Llanos, 1998-2004. Universidad de los Llanos, Villavicencio, Meta., 2006.

24. Ministerio de Educación Nacional MEN, 2013. Disponible en: http://www. mineducacion.gov.co/sistemasdeinformacion/1735/articles-254702_archivo_pdf_ estadisticas_2013.pdf

25. L. Cabrera, J. Tomás, P. Álvarez \& M. González, "The Dropout Problem in University Study", Revista Electrónica de Investigación y Evaluación Educativa (Relieve), vol. 2, 2006.

26. S. R. Meléndez., y M. D. Meriño, Estudio sobre deserción y permanencia académica en la Facultad de Ingeniería de la Universidad de la Guajira desde el II PA 2005 hasta el II PA 2007. Programa de Ingeniería Industrial, Facultad de Ingeniería, Universidad de la Guajira, 2008.

27. Universidad Nacional de Colombia, Cuestión de Supervivencia: Graduación, deserción y rezago en la Universidad Nacional de Colombia, primera edición. Di- rección Nacional de Bienestar Universitario, Universidad Nacional de Colombia, Bogotá (Colombia), 2007.

28. Universidad Abierta para Adultos UAPA, Deserción en las instituciones de educación superior a distancia en América Latina y el Caribe. Ediciones UAPA, Santiago, Republica Dominicana, 2009.

29. L.A. Patiño., y Z.R. Reyes, Factores asociados con la deserción de los estudiantes del programa de Licenciatura en Matemáticas semestre B Tesis de Especialización en Docencia Universitaria, Facultad de Educación, Universidad de Nariño. San Juan de Pasto, Nariño (Colombia), 2004.

30. J.M. Hernández-Mata, R. Hernández-Castro, A. Nieto-Caraveo., y J.F. Hernández-Sierra, "Factores de riesgo para la deserción de estudiantes en la Facultad de Medicina de la Universidad Autónoma de San Luis Potosí (UASLP)", Revista Gaceta 71 Médica de México, vol. 141, 2005.

31. Secretaría de Educación Departamental del Caquetá - SED Caquetá, Nuestros Centros e Instituciones Educativas, 2016. Disponible en: http://www.sedcaqueta. gov.co/nuestrasinstituciones.html, consultado el 11.02.2016

32. G. Olave-Arias, L. García-Rojas., y M. Cisneros-Estupiñan, "Deserción universitaria y alfabetización académica”, Revista Educación y Educadores, vol. 16, 2013.

33. M. J. Martínez A.A. Ortega., y F. Y. Martínez, La problemática actual de la deserción escolar, un análisis desde lo local. Facultad de Economía Internacional, Universidad Autónoma de Chihuahua, Chihuahua (México), 2011.

34. F.A. Barrios, (2013). “Deserción Universitaria en Chile: Incidencia del Financiamiento y otros Factores Asociados", Revista Centro de Investigación Social (CIS), 2013.

35. O. Espinoza, D. Castillo, L. González., y J. Loyola, "Factores familiares asociados 
a la deserción escolar en Chile", Revista de Ciencias Sociales, vol. 18, 2012.

36. LF. Londoño, (2013). "Factores de riesgo presentes en la deserción estudiantil en la Corporación Universitaria Lasallista", Revista Virtual Universidad Católica del Norte, vol. 38, 2013. Número 1.

37. C. M. Román, (2013). “Factores asociados al abandono y la deserción escolar en América Latina: una mirada en conjunto", Revista Iberoamericana sobre Calidad, Eficacia y Cambio en Educación, vol. 11, 2013.

38. L. Alcázar, "Asistencia y Deserción en Escuelas Secundarias Rurales del Perú", Revista Iberoamericana sobre Calidad, Eficacia y Cambio en Educación, vol. 7, 2009.

39. E.J. Duque., y CR. Chaparro, (2012).

"Medición de la percepción de la calidad del servicio de educación por parte de los estudiantes de la UPTC Duitama", Revista Criterio Libre, vol. 10, 2012.

40. C. V Sánchez, C.A. Gómez., y P. L. Ramón, "Educación superior y los planes del gobierno, un tema para estudiar", Revista UNIMAR, vol. 34, 2016.

41. B. M. Segura, "Competencias Personales del Docente", Revista Ciencias de la Educación, vol. 2, 2005.

42. L.A Melo, J.E. Ramos., y P.O. Hernández, "La Educación Superior en Colombia: Situación Actual y Análisis de Eficiencia", Borradores de Economía, núm. 808, 2014.

43. G. M. Guevara, "La condición humana del docente", La Tarea, revista de educación y cultura de la sección 47 del SNTE, $n^{\circ} .2,2009$.

44. W. De Vries., A P. León., JF. Romero y S. I. Hernández, “¿Desertores o decepcionados? Distintas causas para abandonar los estudios universitarios", Revista de la Educación Superior, vol. 40,2011.

45. M. Herrera, "El valor de la Escuela y el Fracaso Escolar", La Tarea, revista de edu- cación y cultura de la sección 47 del SNTE, $n^{\circ} .2,2009$.

46. M. Román, (2009) “El Fracaso Escolar de los Jóvenes en la Enseñanza Media ¿Quiénes y por qué Abandonan Definitivamente el Liceo en Chile?", La Tarea, revista de educación y cultura de la sección 47 del SNTE, n. 2, 2009.

47. C.A. Gómez, C. V. Sánchez., y M.A. Rincón. "Análisis del Sistema Institucional de Evaluación de Estudiantes: El caso de la IE Nueva Jerusalén", Revista Cultura Educación y Sociedad, vol. 6, pp. 139-157, 2015.

48. J. Ojanen, La educación en Finlandia. Ministerio de Asuntos Exteriores, Departamento de Prensa y Cultura. Helsiniki (Finlandia), 2002.

49. A. G, Sánchez S. W., y Navarro A.D. García, Factores de Deserción Estudiantil en la Universidad Surcolombiana sede Neiva (2002-2005). Facultad de Educación, Universidad Surcolombiana, Neiva (Huila), 2009.

50. Programa de la Naciones Unidas para el Desarrollo-PNUD. Estos son los retos educativos de Colombia en el año 2015. Disponible en htpp://http://www.colombiaaprende.edu.co/html/home/1592/article-87521.html. Fecha de consulta: febrero 20 de 2015.

51. R. Sandoval, Ausencia de futuro: la juventud colombiana, Revista CEPAL, n. 29, 1986. 


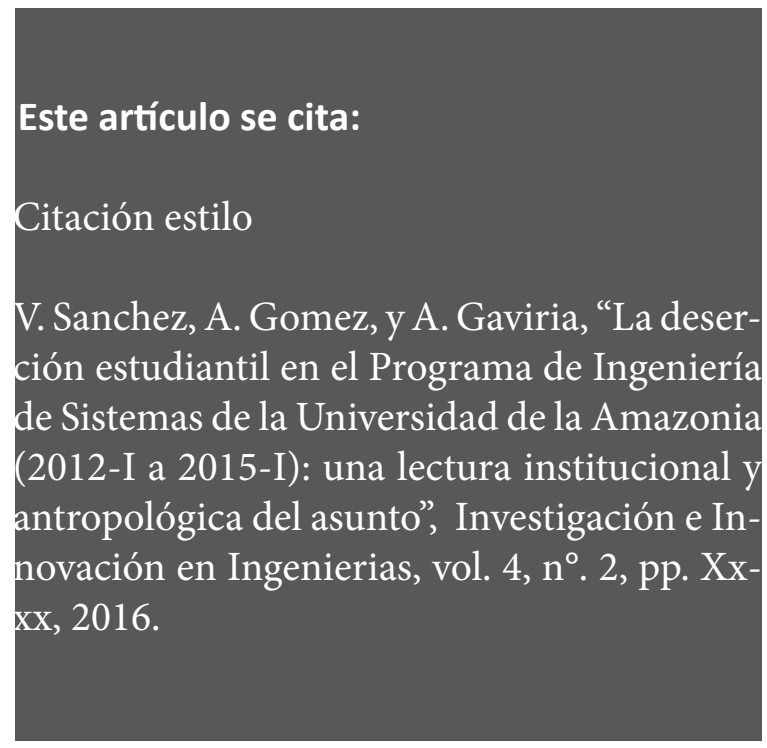

